\title{
$E C L$ : an exhaustive search tool for the identification of cross-linked peptides using whole database
}

\author{
Fengchao $\mathrm{Yu}^{1}$, Ning $\mathrm{Li}^{2^{*}}$ and Weichuan $\mathrm{Yu}^{1,3^{*}}$
}

\begin{abstract}
Background: Chemical cross-linking combined with mass spectrometry (CX-MS) is a high-throughput approach to studying protein-protein interactions. The number of peptide-peptide combinations grows quadratically with respect to the number of proteins, resulting in a high computational complexity. Widely used methods including xQuest (Rinner et al., Nat Methods 5(4):315-8, 2008; Walzthoeni et al., Nat Methods 9(9):901-3, 2012), pLink (Yang et al., Nat Methods 9(9):904-6, 2012), ProteinProspector (Chu et al., Mol Cell Proteomics 9:25-31, 2010; Trnka et al., 13(2):420-34, 2014) and Kojak (Hoopmann et al., J Proteome Res 14(5):2190-198, 2015) avoid searching all peptide-peptide combinations by pre-selecting peptides with heuristic approaches. However, pre-selection procedures may cause missing findings. The most intuitive approach is searching all possible candidates. A tool that can exhaustively search a whole database without any heuristic pre-selection procedure is therefore desirable.
\end{abstract}

Results: We have developed a cross-linked peptides identification tool named ECL. It can exhaustively search a whole database in a reasonable period of time without any heuristic pre-selection procedure. Tests showed that searching a database containing 5200 proteins took $7 \mathrm{~h}$.

ECL identified more non-redundant cross-linked peptides than xQuest, pLink, and ProteinProspector. Experiments showed that about $30 \%$ of these additional identified peptides were not pre-selected by Kojak. We used protein crystal structures from the protein data bank to check the intra-protein cross-linked peptides. Most of the distances between cross-linking sites were smaller than $30 \AA$.

Conclusions: To the best of our knowledge, ECL is the first tool that can exhaustively search all candidates in cross-linked peptides identification. The experiments showed that ECL could identify more peptides than $\times Q$ uest, pLink, and ProteinProspector. A further analysis indicated that some of the additional identified results were thanks to the exhaustive search.

Keywords: Cross-linking, Peptide identification, Database searching

\section{Background}

Chemical cross-linking combined with mass spectrometry (CX-MS) is becoming a powerful approach to studying protein-protein interactions. In the CX-MS protocol, proteins are linked before digestion. Digested products include cross-linked peptides and conventional linear peptides. In this paper, we refer to conventional linear

\footnotetext{
*Correspondence: boningli@ust.hk;eeyu@ust.hk

${ }^{2}$ Division of Life Science, The Hong Kong University of Science and

Technology, Hong Kong, China

${ }^{1}$ Division of Biomedical Engineering, The Hong Kong University of Science and Technology, Hong Kong, China

Full list of author information is available at the end of the article
}

peptides as peptides if there is no ambiguity. Crosslinked peptides are two peptides linked by a chemical compound. Two such peptides are referred to as chains, and the chemical compound is referred to as cross-linker. In the database searching based identification framework, the number of all possible peptide-peptide combinations grows quadratically with respect to the number of proteins, which results in a large search space.

Many tools have been developed to identify crosslinked peptides. An incomplete list includes ASAP [1], MS2Assign [2], MS-Bridge [3], CLPM [4], GPMAW [5], Virtual-MSLab [6], XDB [7], X!Link [8], Popitam [9], MS3D [10], CrossSearch [11], xComb [12], crux 
[13], Xlink-Identifier [14], pLink [27], Hekate [15], ProteinProspector [28, 29], Crossfinder [16], and Kojak [30]. The approach of most of these tools is to modify conventional peptide identification tools' workflow and the corresponding score functions based on the property of cross-linked peptides. Because the search space is large, most of them pre-select high possibility candidates before scoring PSMs (peptide spectrum matches). In order to reduce the search space, cleavable cross-linkers [17-20] have been developed to avoid generating peptide-peptide combinations during database searching. Peptides linked by this kind of crosslinker can be broken into two peptides in dissociation. Thus, the cross-linked peptides identification problem is converted to the conventional peptide identification problem.

Due to the good chemical and biological properties of noncleavable amine-reactive cross-linkers (e.g. DSS (disuccinimidyl suberate) and BS3 (bis(sulfosuccinimidyl) suberate)), they have been widely used recently [21-24]. Tools including xQuest [25, 26], pLink [27], ProteinProspector [28, 29], and Kojak [30] were proposed to identify peptides linked by this kind of cross-linkers. They use preprocessing procedures to eliminate candidates with low possibilities before scoring. Given a spectrum, they compare it with the theoretical spectra from peptides to determine their chances of resulting in high scores heuristically. Peptides with low chances are eliminated. Eliminating some of the peptides before PSM scoring may result in false negatives. The most intuitive approach is searching all candidates exhaustively.

In this paper, we propose a new tool, named ECL (exhaustive cross-linked peptides identification), that can exhaustively search a whole database within a reasonable period of time. Experiments showed that more cross-linked peptides were identified thanks to exhaustive searching. For the purpose of visualization, we developed another tool, named ECLAnnotator, that converts ECL results into webpages. These webpages show annotated tandem mass spectra and matched/unmatched theoretical ions clearly.

\section{Implementation}

ECL is designed to identify peptides linked by noncleavable amine-reactive cross linkers like DSS and BS3. In the current version, ECL only supports CID (collisioninduced dissociation). Given a peptide-peptide combination, ECL in silico fragments it to b-ions and y-ions with different charges. These ions form a theoretical spectrum whose peaks' intensities are the numbers of ions with the corresponding mass-to-charge ratios. The tandem mass spectra produced by a mass spectrometer are referred to as experimental spectra in this paper. ECL uses the normalized cross correlation coefficient to measure the similarity between a theoretical spectrum and an experimental spectrum:

$$
\text { score }=\frac{X^{T} Y}{\|X|\||| Y\|},
$$

where $X$ is the theoretical spectrum, $Y$ is the experimental spectrum, and $T$ stands for vector transpose.

Because the search space is large, we developed an efficient and low memory requirement algorithm to score PSMs. Concretely, Eq. (1) can be rewritten as:

$$
\text { score }=\frac{\left(X_{1}+X_{2}\right)^{T} Y}{\|X|\||| Y\|}=\frac{X_{1}^{T} Y+X_{2}^{T} Y}{\|X|\|| \mid Y}=\frac{X_{1}^{T} \tilde{Y}+X_{2}^{T} \tilde{Y}}{\|X\|},
$$

where $X_{1}$ is the vector whose elements are contributed by the first chain, $X_{2}$ is the vector whose elements are contributed by the second chain, $X_{1}+X_{2}=X$, and $\tilde{Y}=$ $Y /\|Y\|$. ECL calculates $\tilde{Y}$ before scoring PSMs, which reduces the computational complexity largely. Both $X_{1}$ and $X_{2}$ have linear ions containing one chain's amino acids and cross-linking ions containing both chains' amino acids (Fig. 1). Given an experimental spectrum and a chain, ECL can obtain this chain's ion masses as

$$
x_{i}=\left\{\begin{array}{ll}
p-c+l_{i}, & \text { cross }- \text { linking ion } \\
l_{i}, & \text { linear ion }
\end{array},\right.
$$

where $i$ is the ion index starting from $0, x_{i}$ is $i$ th ion's mass, $p$ is the experimental spectrum's precursor mass, $c$ is the chain's mass, and $l_{i}$ is the corresponding linear ion's mass. Taking the first chain in Fig. 1 for example, 4th b-ion is a cross-linking ion containing "EAKE" and "EVRKELDDLR" linked by a cross-linker. Thus, its corresponding linear b-ion is "EAKE". Clearly, $p-c$ is equal to the summation of the other chain's mass and the crosslinker's mass. We don't consider the difference between the experimental spectrum's precursor mass and the theoretical spectrum's precursor mass because the precursor mass tolerance is smaller than or equal to the tandem mass tolerance for almost all mass spectrometers. Given

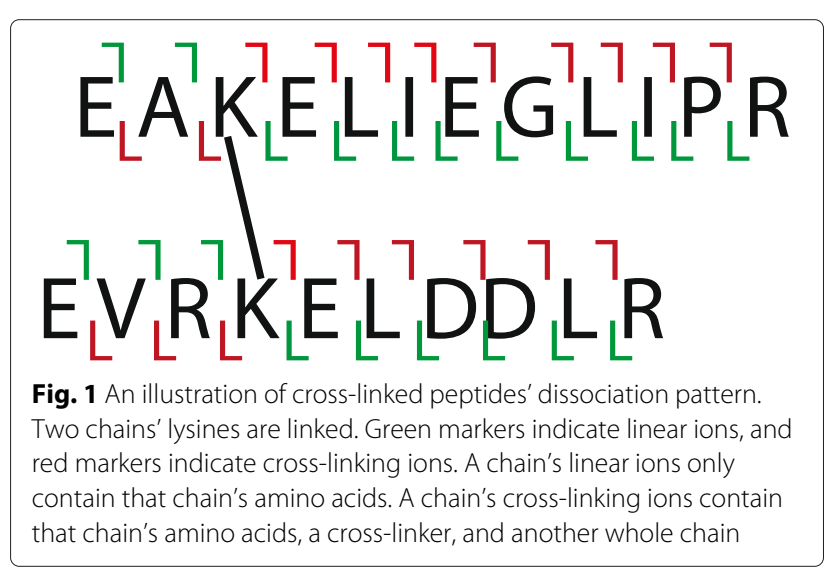


each ion's mass, ECL calculates its corresponding massto-charge ratios with different charges. After getting all ions' mass-to-charge ratios for one chain, ECL generates $X_{1}$ or $X_{2}$. Given an experimental spectrum, $X_{1}^{T} \tilde{Y}$ only needs to be calculated once for different $X_{2}$, which reduces the computational complexity largely.

With the above optimization, ECL's workflow is described as follows:

1. Indexing chains based on their masses.

2. Calculating ions' masses for each chain.

3. Indexing experimental spectra based on their precursor masses.

4. Peak de-noising. Eliminating peaks whose intensities have the highest frequency.

5. Calculating $\tilde{Y}=Y /\|Y\|$ for each experimental spectrum.

6. Finding the largest precursor mass from all experimental spectra.

7. Looping over all chains whose masses are smaller than or equal to half of the largest precursor mass in ascending order:

7.1 Finding all spectra whose precursor masses are larger than or equal to $2 \times c+r-o$, where $r$ is the cross-linker's mass and $o$ is the precursor mass tolerance.

7.2 Calculating ions' masses using Eq. (3), and using these masses to generate $X_{1}$.

7.3 Calculating $X_{1}^{T} \tilde{Y}$ for each corresponding spectrum.

7.4. Finding all chains whose masses are within the range $[p-o-c-r, p+o-c-r)$.

7.5 Looping over all found chains:

7.5.1 Calculating ions' masses using Eq. (3), and using these masses to generate $X_{2}$.

7.5.2 Calculating $X_{2}^{T} \tilde{Y}$.

7.5.3 Calculating the final score using Eq. (2).

7.5.4 Saving each spectrum's top score result as a PSM.

8. Estimating FDR (false discovery rate) for each PSM.

9. Converting FDR to $q$-value.

ECL estimates FDR as what xProphet [26] and pLink [27] do. Three kinds of PSMs are used:

1. Both chains are from the target database.

2. Both chains are from the decoy database.

3. One chain is from the target database and the other chain is from the decoy database.

FDR is estimated with

$$
\operatorname{FDR}(s)=\frac{f(s)-d(s)}{t(s)},
$$

where $s$ is a score, $t(s)$ is the number of the first kind of PSMs whose scores are smaller than or equal to $s, d(s)$ is the number of the second kind of PSMs whose scores are smaller than or equal to $s$, and $f(s)$ is the number of the third kind of PSMs whose scores are smaller than or equal to $s$. Finally, FDR is converted to $q$-value [31]:

$$
q(t)=\min _{s \leq t} F D R(s),
$$

where $t$ is a threshold.

\section{Results and discussion \\ Computational complexity analysis}

ECL is closely related to the work of Chen et al. [32] and Kojak [30]. Chen et al. [32] provided their algorithm's computational complexity. Hoopmann et al. [30] provided Kojak's source code without computational complexity analysis, so we analyzed its computational complexity based on the source code. In this section, we will analyze ECL's computational complexity in detail.

\section{Computational complexity analysis}

Defining the following variables:

- $k$ : number of proteins in a database.

- $n$ : average number of peptides in a protein.

- $m$ : average length of a chain.

- $h$ : average number of peaks in an experimental spectrum.

- $s$ : number of experimental spectra.

- $L$ : number of precursor mass tolerance ranges. This approximately equals the precursor mass range divided by the precursor mass tolerance.

The time complexity of the algorithm proposed by Chen et al. [32] is

$$
O\left(s k n^{2} \log (k n)+s k^{2} n^{2} \log (k n) / L+s k^{2} n^{2}(m+h) / L\right) .
$$

For the first and second terms, the authors only considered one experimental spectrum. We multiply the terms by $s$ because there are $s$ experimental spectra. We also use $k^{2} n^{2} / L$ to replace $p$ in the original paper. For the third term, the authors only considered one PSM. We multiply the term by $s k^{2} n^{2} / L$ because there are $k^{2} n^{2} / L$ peptidepeptide combinations for each experimental spectrum and there are $s$ experimental spectra. The time complexity of Kojak is

$$
O\left(k n \log (s)+k n s(m+h+1)+s t^{2}\right) .
$$

Please refer to the Additional file 1 for details.

For ECL, the computational complexity is dominated by step 7 in the workflow. The complexity of step 7.1 is $O(\log (s))$. Steps 7.2 and 7.5.1 have the same time complexity, $O(m)$. ECL stores theoretical and experimental spectra 
in sparse matrixes. We developed an algorithm to match peaks between a theoretical spectrum and an experimental spectrum with $O(m+h)$ complexity (Algorithm 1$)$. Thus, both steps 7.3 and 7.5.2 have the time complexity, $O(m+h)$. Moreover, for an experimental spectrum and a pair of chains, steps 7.2 and 7.3 only need to be executed once because ECL checks each chain whose mass is smaller than or equal to half of the largest precursor mass in ascending order. Steps 7.3 and 7.5.2 also only need to be executed once for the same reason. The time complexity of step 7.4 is $O(\log (k n))$. The time complexity of steps 7.5.3 and 7.5.4 is $O(k n s / L)$. Thus, the time complexity of step 7 is

$$
O(k n(\log (s)+m+s(m+h)+\log (k n)+k n s / L)) .
$$

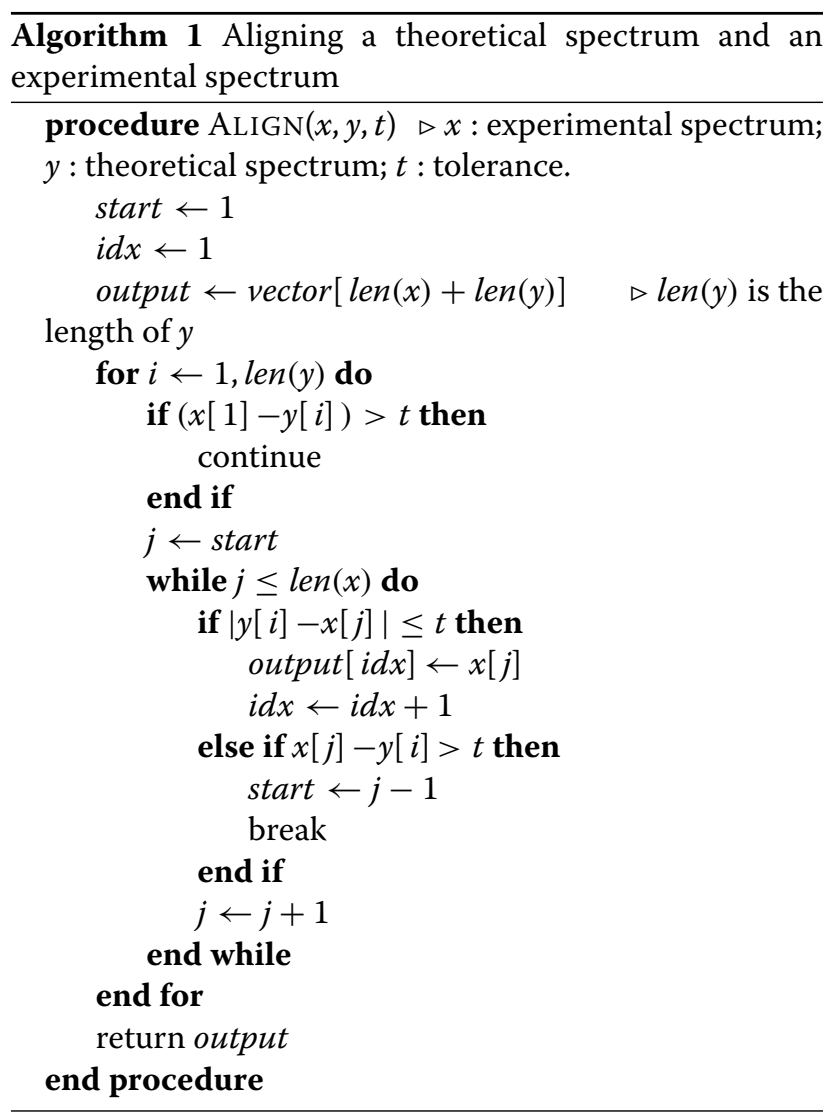

There are seven variables in the time complexity equations. Five of them can be fixed based on biological prior knowledge:

- $n \approx 100$.

- $m \approx 20$.

- $h \approx 10^{2}$.

- $s \approx 10^{4}$.

- $L \approx 10^{5}$.
We plotted curves of Eqs. (6), (7), and (8) against different numbers of proteins (Fig. 2). Since Kojak selects $t$ peptides for each spectrum, we plotted three curves corresponding to three different $t$ values. We can see that Chen et al. [32] has the highest time complexity. When the number of proteins is small, ECL has smaller time complexity compared to Kojak (leftmost of Fig. 2). This is because ECL doesn't need to select peptides beforehand. When the number of protein is large, ECL has higher complexity than Kojak (rightmost of Fig. 2). This is because the number of peptide-peptide combinations searched by ECL grows quadratically as the increase of protein number (Eq. (8)). This is an unavoidable cost of exhaustive searching. On the other hand, the number of peptide-peptide combinations searched by Kojak is almost constant, and the total time complexity increases linearly (Eq. (7)).

Even though ECL's time complexity is large, it can still handle a large database. Given a data set containing thousands of tandem mass spectra, ECL only needs $7 \mathrm{~h}$ to search a database containing 5200 proteins.

\section{Space complexity}

- The space complexity of Chen et al. [32] is

$$
O\left(k n+k^{2} n^{2} / L+k n m+h\right) .
$$

For the second term, we use $k^{2} n^{2} / L$ to replace $p$ in the original paper. For the third term, the authors only considered one peptide-peptide combination for each experimental spectrum. We multiply the term by $k n$ considering that there are $k n$ peptides for each experimental spectrum.

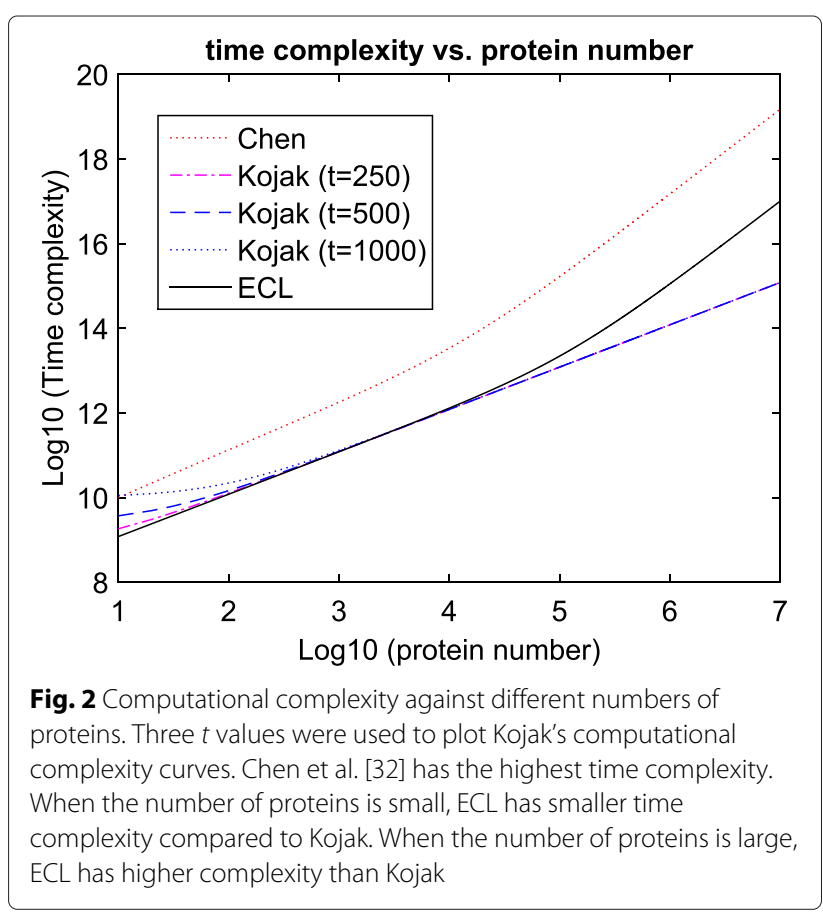


- There are two steps in Kojak. The space complexity of the first step is $O(m+s h)$, and the space complexity of the second step is $O(t m+h)$. Thus, the total space complexity is

$$
O(m+s h+t m+h) \text {. }
$$

- The space complexity of ECL is

$$
O(k n m+s h) \text {. }
$$

Clearly, Chen et al. [32] has the highest space complexity, and Kojak has the lowest space complexity. Although ECL's space complexity is higher than that of Kojak, from our experience, a personal computer with $32 \mathrm{G}$ memory is sufficient in most cases.

\section{Experiments}

In this paper, we will present two sets of experiments. The first one used a data set from the cross-linking of two synthetic peptides. The second one used four data sets from the $26 \mathrm{~S}$ proteasome sample [33] provided by $x$ Quest $[25,26]$. Since our study did not involve any humans, animals or clinical data, we do not have ethics or consent issues.

\section{An experiment with synthetic peptides}

This experiment used two synthetic peptides produced by GL Biochem (Shanghai) Ltd. The sequences were "EVRKELDDLR" and "EAKELIEGLPR". N-terminals were protected by Fmoc. We used $1 \mu \mathrm{L}$ peptides and $0.5 \mu \mathrm{L}$ DSS. Their concentrations were 1 and $0.5 \mathrm{mM}$, respectively. We dissolved the peptides and DSS in DMSO (dimethyl sulfoxide) to a final concentration of $50 \mathrm{mM}$. The reaction was carried out at room temperature, and the reaction time was $2 \mathrm{~h}$. After quenching, we added $12.5 \mu \mathrm{L}$ piperidine to the above solution to remove the Fmoc protection. The reaction lasted for another $2 \mathrm{~h}$. Finally, we freeze-dried the sample to obtain the crosslinked peptides.

LC-MS (liquid chromatography-mass spectrometry) analysis was carried out on a Thermo LTQ Orbitrap XL mass spectrometer (Thermo Fisher Scientific Inc.) with a NanoLC system. The sample was loaded onto a trapping column (PepMap C18; $2 \mathrm{~cm} \times 100 \mu \mathrm{m} \times 5 \mu \mathrm{m}, 100 \AA$ ) using a flow rate of $4 \mu \mathrm{L} / \mathrm{min}$ of solvent $\mathrm{A}$. The loading lasted for $10 \mathrm{~min}$. Cross-linked peptides were separated at a flow rate of $200 \mathrm{~L} / \mathrm{min}$ on a $75 \mu \mathrm{m} \times 50 \mathrm{~cm} \mathrm{C18} \mathrm{column}$ (Acclaim PepMap RSLC C18, $75 \mu \mathrm{m} \times 50 \mathrm{~cm} \times 3 \mu \mathrm{m}$, $100 \AA$ ). The following gradient was used: $0-8 \min 2 \% \mathrm{~B}$, 8-12 min 2-10\% B, 12-180 min 10-50\% B, 180-200 min 50-98 \% B, 200-215 min $98 \%$ B, and 215-240 min $98-$ $2 \% \mathrm{~B}$, where $\mathrm{B}$ was the ratio of acetonitrile to formic acid. B equaled 100:0.1 in this experiment. The mass spectrometer selected up to five precursors to perform CID. The intensity threshold of triggering fragmentation was 150 counts. Only those whose precursor charges were larger than or equal to 2 were considered. CID was performed for $30 \mathrm{~ms}$ using $35 \%$ normalized collision energy and a 0.25 activation value. Dynamic exclusion was used with the following parameters: 1 repeat count, $60 \mathrm{~s}$ exclusion duration, 500 list size, and $10 \mathrm{ppm}$ mass window. The ion target value was $1,000,000$ (or $500 \mathrm{~ms}$ fill time) for full scans, and 1,000,000 (or $200 \mathrm{~ms}$ fill time) for a tandem mass scan. Fragmented ions were detected in a linear ion trap.

During the search, the precursor mass tolerance was $10 \mathrm{ppm}$, and the tandem mass tolerance was $0.5 \mathrm{Th}$. Up to 2 missed cleavages were allowed. The database contained 100 randomly selected proteins and two synthetic peptides. The decoy database was generated by reversing peptides, with lysine and arginine fixed. Because there was only one linkable site in each synthetic peptide, all crosslinked peptides formed by synthetic peptides were treated as inter-protein cross-linked peptides. The $q$-value cut-off threshold was 0.05 .

The search was carried out on a personal computer with an Intel Core i5-4570 CPU (central processing unit) and $32 \mathrm{~GB}$ memory. ECL needed about $100 \mathrm{~s}$ to finish the task. Since we knew the ground truth, we could calculate the false discovery proportion. 4 out of 149 PSMs were incorrect. The corresponding false discovery proportion was 0.03 . This experiment indicated that ECL could provide trustable results. Details can be found in the Additional file 2.

\section{Experiments with 265 proteasome data}

Four data sets from the $26 \mathrm{~S}$ proteasome sample $[25,26,33]$ were used. We first searched four data sets against a database released along with the data sets. It contained 34 proteins. The latest versions of xQuest, pLink, ProteinProspector, Kojak, and ECL were used: xQuest 2.1.1, pLink 1.23, ProteinProspector 5.14.4, Kojak 1.4.2, and ECL 20160117. The precursor mass tolerance was $10 \mathrm{ppm}$, and the tandem mass tolerance was $0.2 \mathrm{Da}$. Other parameters were the same as those in the previous experiment. All the parameter files used by these tools were included in the Additional file 3. We used xProphet [26] to estimate the $q$ value for $x Q u e s t$ 's results by setting "qtransform" to 1 in the "xproph.def" file. Because ProteinProspector did not provide the $q$-value in its results, we estimated it as what Trnka et al. [29] did. We used Percolator to estimate the $q$-value for Kojak's results as what Kojak required. Intra-protein cross-linked peptides and interprotein cross-linked peptides were analyzed separately. For a fair comparison, these tools' $q$-value thresholds were 0.05 .

Table 1 shows the numbers of non-redundant cross-linked peptides identified by xQuest, pLink, 
Table 1 Numbers of non-redundant cross-linked peptides identified by xQuest, pLink, ProteinProspector, Kojak, and ECL, respectively. The database contains 34 proteins

\begin{tabular}{llllll}
\hline Data set & xQuest & pLink & ProteinProspector & Kojak & ECL \\
\hline 1 & $70(56)$ & $5(4)$ & $104(69)$ & $102(71)$ & 97 \\
2 & $73(41)$ & $28(17)$ & $99(45)$ & $120(56)$ & 58 \\
3 & $90(62)$ & $28(10)$ & $96(64)$ & $139(90)$ & 127 \\
4 & $61(47)$ & $20(14)$ & $94(68)$ & $110(83)$ & 135 \\
\hline
\end{tabular}

Values in the brackets are the numbers of overlapping cross-linked peptides identified by both ECL and the corresponding method

ProteinProspector, Kojak, and ECL, respectively. Corresponding Venn diagrams can be found in the Additional file 1. ECL identified more cross-linked peptides than xQuest, pLink, and ProteinProspector. We used protein crystal structures from the protein data bank (PDB) to measure the distances between linking-sites in intra-protein cross-linked peptides. Only 3 proteins had structural information. Their UniProt accessions were O94444, P06732, and P50524, respectively. The corresponding PDB ID were $2 \mathrm{X} 5 \mathrm{~N}, 1 \mathrm{IOE}$, and $4 \mathrm{~B} 0 \mathrm{Z}$, respectively. There were 65 PSMs to these proteins. 60 of them had a distance smaller than $30 \AA$, which meant that they were within the distance tolerance. Details can be found in the Additional file 4. We also used ECLAnnotator to generate annotated tandem mass spectra for ECL's results. They can be found at http://bioinformatics.ust. hk/ecl.html. Then, we analyzed matched and unmatched peaks. Please refer to the Additional file 2 for details.

In order to find out if the additionally identified cross-linked peptides were due to exhaustive search, we let Kojak output top 9999 pre-selected peptides for each cross-linked peptide's highest score spectrum. (The default number of pre-selected peptides is 250 . To our knowledge, other tools can not output their pre-selected peptides). Then, we compared the cross-linked peptides identified by ECL with those pre-selected peptides in the corresponding spectra. We consider one additionally identified cross-linked peptides pair is due to exhaustive search if all of the following criteria are satisfied (We thank the anonymous reviewer for suggesting these criteria):

1. The precursor masses in Kojak and ECL are within the same tolerance range.

2. If both of two peptide chains are in the pre-selection list and at least one is over 250, Kojak and ECL identify the same pair of peptide chains.

3. At least one peptide chain isn't in the pre-selection list.

Table 2 shows the summarized results. About $30 \%$ of these peptides aren't within top 250 of Kojak's pre-selected peptides, which means that the pre-selection procedure is one of the causes of missing findings. Each spectrum's pre-
Table 2 A table showing if Kojak searched those missing identified peptides

\begin{tabular}{llll}
\hline Data set & $\begin{array}{l}\text { Number of peptides from } \\
\text { the cross-linked peptides } \\
\text { identified by ECL, but not } \\
\text { by Kojak }\end{array}$ & $\begin{array}{l}\text { Number of peptides } \\
\text { that don't belong to } \\
\text { Kojak's pre-selected } \\
\text { peptides }\end{array}$ & Ratio \\
\hline 1 & 25 & 2 & 0.08 \\
2 & 2 & 1 & 0.50 \\
3 & 37 & 12 & 0.32 \\
4 & 52 & 21 & 0.40 \\
Total & 116 & 36 & 0.31 \\
\hline
\end{tabular}

The second column contains the total numbers of peptides from the cross-linked peptides identified by ECL, but not by Kojak. The third column contains the numbers of peptides that don't belong to Kojak's pre-selected peptides. The forth column contains the ratios between the number in the third column and the number in the second column

selected peptides and detailed comparison results can be found in the Additional file 5.

Table 3 shows the corresponding running time of xQuest, pLink, Kojak, and ECL, respectively. ProteinProspector spent 1254 seconds on average analyzing one data set. It was run on the authors' web server so we didn't compare it with the other four tools. Since Kojak supports multi-thread computing, we ran it with 4 threads. xQuest, pLink, and ECL don't support multi-thread computing.

Finally, we tested if ECL could search a large database within a reasonable period of time. We searched the same data sets against the whole proteome of Schizosaccharomyces pombe species. There were 5200 proteins. We set the allowed maximum missed cleavage to 1 . The rest of the parameters were the same as those in the last experiment. xQuest ran for a few days, but it still couldn't finish the searching. pLink could not handle such a large database. ProteinProspector spent $1.7 \mathrm{~h}$ on average analyzing one data set on the authors' web server. Kojak spent $0.25 \mathrm{~h}$ on average analyzing one data set. ECL spent $7 \mathrm{~h}$ on average analyzing one data set.

There were $4 \times 10^{10}$ peptide-peptide combinations including decoy peptides. The precursor mass tolerance was $10 \mathrm{ppm}$. Thus, there were about $4 \times 10^{5}$ peptidepeptide combinations for each spectrum. Kojak selected top 250 peptides to generate peptide-peptide combinations for each spectrum, which covered about $8 \%$ of the whole search space. ProteinProspector used a similar preselection procedure to select top 1000 peptides. Thus,

Table 3 Running time of xQuest, pLink, Kojak, and ECL, respectively. The unit is second

\begin{tabular}{lllll}
\hline Data set & xQuest & pLink & Kojak (4 threads) & $\mathrm{ECL}$ \\
\hline 1 & 6349 & 851 & 46 & 51 \\
2 & 6741 & 878 & 48 & 57 \\
3 & 20419 & 876 & 49 & 60 \\
4 & 21757 & 700 & 47 & 60 \\
\hline
\end{tabular}


the number of peptide-peptide combinations searched by ProteinProspector and Kojak was almost constant with the increase of the database size. However, the number of peptide-peptide combinations searched by ECL increased quadratically. That's why ECL was slower than ProteinProspector and Kojak.

ProteinProspector, Kojak, and ECL identified fewer cross-linked peptides compared with the previous experiment (Table 4). It is a known issue [34, 35] that larger databases lead to fewer results. The discussion of this issue is beyond the scope of this paper. ECL identified more non-redundant peptides than ProteinProspector and Kojak. Please note that there is no intra-protein crosslinked peptides identified by Kojak because Percolator output errors in estimating $q$-value for Kojak. The errors said: "the input data has too good separation between target and decoy PSMs". It is a common error when there are only a few target or decoy PSMs. Please refer to Percolator's document for more detail.

\section{Conclusions}

High computational complexity is a major obstacle in exhaustively carrying out large-scale cross-linked peptides identification. To the best of our knowledge, ECL is the first tool that successfully addresses the computational complexity issue without any heuristic pre-selection procedure. Given thousands of tandem mass spectra and a database containing thousands of proteins, it can finish the task in a few hours. The experiments showed that ECL could identify more peptides than $x Q u e s t$, pLink, and ProteinProspector. A further analysis on public data sets showed that exhaustive search helped identify more cross-linked peptides than existing methods.

\section{Availability and requirements \\ Project name: ECL}

Project home pase: http://bioinformatics.ust.hk/ecl.html Operating systems: Windows, Linux, OS X

Programming language: Java, Python

Other requirements: Java 1.7 or higher, Python 2.7

License: Apache License 2

Table 4 Numbers of non-redundant cross-linked peptides identified by ProteinProspector, Kojak, and ECL, respectively. The database contains 5200 proteins

\begin{tabular}{llll}
\hline Data set & ProteinProspector & Kojak & $\mathrm{ECL}$ \\
\hline 1 & $20(15)$ & $5(0)$ & 36 \\
2 & $32(16)$ & $6(0)$ & 39 \\
3 & $24(12)$ & $4(0)$ & 39 \\
4 & $23(17)$ & $2(0)$ & 57
\end{tabular}

Values in the brackets are the numbers of overlapping cross-linked peptides identified by both ECL and the corresponding method. There is no result for intra-protein cross-linked peptides reported by Kojak because Percolator outputs errors in estimating $q$-value

\section{Additional files}

Additional file1: A supplementary document contains ECL user instruction, computational complexity analysis of Kojak, spectra analysis of the 265 Proteasome results, and venn diagrams of 265 Proteasome results. (PDF $434 \mathrm{~kb}$ )

Additional file 2: Detailed results of synthetic peptides and 265 Proteasome samples. (ZIP $4432 \mathrm{~kb}$ )

Additional file 3: Parameter files used by $\times$ Quest, ProteinProspector, Kojak, and ECL, respectively. (ZIP $17 \mathrm{~kb}$ )

Additional file 4: Distances of intra protein identified by ECL. (XLSX 15 kb) Additional file 5: Kojak's pre-selection list of PSMs only identified by ECL. (ZIP $5147 \mathrm{~kb})$

\section{Abbreviations}

BS3: Bis(sulfosuccinimidyl) suberate. CID: collision-induced dissociation; CPU: central processing unit; CX-MS: chemical cross-linking combined with mass spectrometry; DMSO: dimethyl sulfoxide; DSS: disuccinimidyl suberate; ECL: exhaustive cross-linked peptides identification tool; ETD: electron-transfer dissociation; FDR: false discovery rate; PDB: protein data bank; PSM: peptide spectrum match.

\section{Acknowledgements}

We would like thank for the anonymous reviewers for all the critical challenges, and excellent suggestions.

\section{Funding}

This work is partially supported by a theme-based project T12-402/13N from the research grant council (RGC) of the Hong Kong S.A.R. government, internal grant VPRGO15EG01 from HKUST, two grants, 16101114 and 661613, from the general research fund (GRF) of the Hong Kong S.A.R. government, and grant 31370315 from the National Natural Science Foundation of China (NSFC).

\section{Availability of data and materials}

The mzXML file of the synthetic peptide sample can be downloaded at http:// bioinformatics.ust.hk/ecl.html. Four mzXML files of the 265 proteasome sample can be found in the XQuest virtual machine from http://proteomics. ethz.ch/cgi-bin/xquest2_cgi/installation.cgi.

\section{Authors' contributions}

FY designed the algorithm, wrote the program, analyzed the computational complexity, did the benchmarks, and wrote the manuscript. NL conceived the study and provided the synthetic peptides sample. WY conceived the study and revised the manuscript. All authors read and approved the final manuscript.

\section{Competing interests}

The authors declare that they have no competing interests.

\section{Consent for publication}

Not applicable.

\section{Ethics approval and consent to participate} Not applicable.

\section{Author details}

${ }^{1}$ Division of Biomedical Engineering, The Hong Kong University of Science and Technology, Hong Kong, China. ${ }^{2}$ Division of Life Science, The Hong Kong University of Science and Technology, Hong Kong, China. ${ }^{3}$ Department of Electronic and Computer Engineering, The Hong Kong University of Science and Technology, Hong Kong, China.

Received: 20 August 2015 Accepted: 7 May 2016

Published online: 20 May 2016

References

1. Young MM, Tang N, Hempel JC, Oshiro CM, Taylor EW, Kuntz ID, Gibson BW, Dollinger G. High throughput protein fold identification by using experimental constraints derived from intramolecular cross-links and mass spectrometry. Proc Natl Acad Sci U S A. 2000;97:5802-806. 
2. Schilling B, Row RH, Gibsonb BW, Guo X, Young MM. MS2Assign: Automated assignment and nomenclature of tandem mass spectra of chemically crosslinked peptides. J Am Soc Mass Spectrom. 2003;14: 834-50.

3. Chu F, Shan S-O, Moustakas DT, Alber F, Egea PF, Stroud RM, Walter P, Burlingame AL. Unraveling the interface of signal recognition particle and its receptor by using chemical cross-linking and tandem mass spectrometry. Proc Natl Acad Sci U S A. 2004;101(47):16454-16459.

4. Tang Y, Chen Y, Lichti C, Hall R, Raney K, Jennings S. CLPM: a cross-linked peptide mapping algorithm for mass spectrometric analysis. BMC Bioinforma. 2005;6:9.

5. Ihling C, Schmidt A, Kalkhof S, Schulz DM, Stingl C, Mechtler K, Haack M, Beck-Sickinger AG, Cooper DM, Sinz A. Isotope-labeled cross-linkers and fourier transform ion cyclotron resonance mass spectrometry for structural analysis of a protein/peptide complex. J Am Soc Mass Spectrom. 2006;17(8):1100-1113.

6. Koning LJ, Kasper PT, Back JW, Nessen MA, Vanrobaeys F, Beeumen J, Gherardi E, Koster CG, Jong L. Computer-assisted mass spectrometric analysis of naturally occurring and artificially introduced cross-links in proteins and protein complexes. FEBS J. 2006;273(2):281-91.

7. Maiolica A, Cittaro D, Borsotti D, Sennels L, Ciferri C, Tarricone C, Musacchio A, Rappsilber J. Structural analysis of multi-protein complexes by cross-linking, mass spectrometry and database searching. Mol Cell Proteomics. 2007;6:2200-211.

8. Lee YJ, Lackner LL, Nunnari JM, Phinney BS. Shotgun cross-linking analysis for studying quaternary and tertiary protein structures. J Proteome Res. 2007;6(10):3908-917.

9. Singh P, Shaffer SA, Scherl A, Holman C, Pfuetzner RA, Freeman TJL, Miller SI, Hernandez P, Appel RD, Goodlett DR. Characterization of protein cross-links via mass spectrometry and an open-modification search strategy. Anal Chem. 2008;80(22):8799-806.

10. Yu ET, Hawkins A, Kuntz ID, Rahn LA, Rothfuss A, Sale K, Young MM Yang CL, Pancerella CM, Fabris D. The collaboratory for MS3D: a new cyberinfrastructure for the structural elucidation of biological macromolecules and their assemblies using mass spectrometry-based approaches. J Proteome Res. 2008;7(11):4848-857.

11. Nadeau OW, Wyckoff GJ, Paschall JE, Artigues A, Sage J, Villar MT, Carlson GM. CrossSearch, a user-friendly search engine for detecting chemically cross-linked peptides in conjugated proteins. Mol Cell Proteomics. 2008;7(4):739-49.

12. Panchaud A, Singh P, Shaffer SA, Goodlett DR. xComb: a cross-linked peptide database approach to protein-protein interaction analysis. J Proteome Res. 2010;9(5):2508-515.

13. Mcllwain S, Draghicescu P, Singh P, Goodlett DR, Noble WS. Detecting cross-linked peptides by searching against a database of cross-linked peptide pairs. J Proteome Res. 2010;9(5):2488-495.

14. Du X, Chowdhury SM, Manes NP, Wu S, Mayer MU, Adkins JN, Anderson GA, Smith RD. Xlink-Identifier: an automated data analysis platform for confident identifications of chemically cross-linked peptides using tandem mass spectrometry. J Proteome Res. 2011;10(3):923-31.

15. Holding AN, Lamers MH, Stephens E, Skehel JM. Hekate: software suite for the mass spectrometric analysis and three-dimensional visualization of cross-linked protein samples. J Proteome Res. 2013;12(12):5923-933.

16. Mueller-Planitz F. Crossfinder-assisted mapping of protein crosslinks formed by site-specifically incorporated crosslinkers. Bioinformatics. 2015;31(12):2043-5.

17. Petrotchenko EV, Borchers CH. ICC-CLASS: isotopically-coded cleavable crosslinking analysis software suite. BMC Bioinforma. 2010;11(1):64.

18. Kao A, Chiu CL, Vellucci D, Yang Y, Patel VR, Guan S, Randall A, Baldi P, Rychnovsky SD, Huang L. Development of a novel cross-linking strategy for fast and accurate identification of cross-linked peptides of protein complexes. Mol Cell Proteomics. 2010;mcp-M110.

19. Petrotchenko EV, Serpa JJ, Borchers CH. An isotopically coded cid-cleavable biotinylated cross-linker for structural proteomics. Mol Cell Proteomics. 2011;10(2):110-001420.

20. Kaake RM, Wang X, Burke A, Yu C, Kandur W, Yang Y, Novtisky EJ, Second $\mathrm{T}$, Duan J, Kao A, et al. A new in vivo cross-linking mass spectrometry platform to define protein-protein interactions in living cells. Mol Cell Proteomics. 2014;13(12):3533-543.

21. Herzog F, Kahraman A, Boehringer D, Mak R, Bracher A, Walzthoeni T, Leitner A, Beck M, Hartl FU, Ban N, et al. Structural probing of a protein phosphatase $2 \mathrm{~A}$ network by chemical cross-linking and mass spectrometry. Science. 2012;337(6100):1348-1352.

22. Nguyen VQ, Ranjan $A$, Stengel $F$, Wei $D$, Aebersold $R$, Wu C, Leschziner AE. Molecular architecture of the ATP-dependent chromatin-remodeling complex SWR1. Cell. 2013;154(6):1220-1231.

23. Politis A, Stengel F, Hall Z, Hernández H, Leitner A, Walzthoeni T, Robinson CV, Aebersold R. A mass spectrometry-based hybrid method for structural modeling of protein complexes. Nat Methods. 2014;11(4):403-6.

24. Greber BJ, Boehringer D, Leitner A, Bieri P, Voigts-Hoffmann F, Erzberger JP, Leibundgut M, Aebersold R, Ban N. Architecture of the large subunit of the mammalian mitochondrial ribosome. Nature. 2014;505(7484):515-9.

25. Rinner O, Seebacher J, Walzthoeni T, Mueller L, Beck M, Schmidt A, Mueller M, Aebersold R. Identification of cross-linked peptides from large sequence databases. Nat Methods. 2008:5(4):315-8.

26. Walzthoeni T, Claassen M, Leitner A, Herzog F, Bohn S, Förster F, Beck M, Aebersold R. False discovery rate estimation for cross-linked peptides identified by mass spectrometry. Nat Methods. 2012;9(9):901-3.

27. Yang B, Wu YJ, Zhu M, Fan SB, Lin J, Zhang K, Li S, Chi H, Li YX, Chen HF, et al. Identification of cross-linked peptides from complex samples. Nat Methods. 2012;9(9):904-6.

28. Chu F, Baker PR, Burlingame AL, Chalkley RJ. Finding chimeras: a bioinformatics strategy for identification of cross-linked peptides. Mol Cell Proteomics. 2010;9:25-31.

29. Trnka MJ, Baker PR, Robinson PJ, Burlingame A, Chalkley RJ. Matching cross-linked peptide spectra: only as good as the worse identification. Mol Cell Proteomics. 2014;13(2):420-34.

30. Hoopmann MR, Zelter A, Johnson RS, Riffle M, MacCoss MJ, Davis TN, Moritz RL. Kojak: efficient analysis of chemically cross-linked protein complexes. J Proteome Res. 2015;14(5):2190-198.

31. Storey JD, Tibshirani R. Statistical significance for genomewide studies. Proc Natl Acad Sci. 2003;100(16):9440-445.

32. Chen T, Jaffe JD, Church GM. Algorithms for identifying protein cross-links via tandem mass spectrometry. In: Proceedings of the fifth annual international conference on Computational biology. ACM; 2001. p. 95-102.

33. Bohn S, Beck F, Sakata E, Walzthoeni T, Beck M, Aebersold R, Frster F, Baumeister W, Nickell S. Structure of the $26 S$ proteasome from schizosaccharomyces pombe at subnanometer resolution. Proc Natl Acad Sci U S A. 2010;107(49):20992-0997.

34. Nesvizhskii Al. A survey of computational methods and error rate estimation procedures for peptide and protein identification in shotgun proteomics. J Proteome. 2010;73(11):2092-123.

35. Kertesz-Farkas A, Keich U, Noble WS. Tandem mass spectrum identification via cascaded search. J Proteome Res. 2015;14(8):3027-38.

\section{Submit your next manuscript to BioMed Central and we will help you at every step:}

- We accept pre-submission inquiries

- Our selector tool helps you to find the most relevant journal

- We provide round the clock customer support

- Convenient online submission

- Thorough peer review

- Inclusion in PubMed and all major indexing services

- Maximum visibility for your research

Submit your manuscript at www.biomedcentral.com/submit
() Biomed Central 